\author{
Proceedings of the \\ $1^{\text {st }}$ International Conference and Exhibition on Future RFID Technologies \\ Eszterhazy Karoly University of Applied Sciences and \\ Bay Zoltán Nonprofit Ltd. for Applied Research \\ Eger, Hungary, November 5-7, 2014. pp. 115-125
}

doi: 10.17048/FutureRFID.1.2014.115

\title{
Intelligent sensor systems for self-optimising production chains
}

\author{
Von René von Lipinski, Markus Richter, Jörg Reiff-Stephan
}

Technische Hochschule Wildau [FH]

\section{Introduction}

Economic production processes increasingly need to be controlled under the perspective of effectiveness and efficiency at the global supplier market. In this aspect the reception and processing of information is considered to be one of the most significant and crucial factors to encourage innovation in the world of industrial production. The Internet of Things/Services/Data (iD3, short for ger.: Internet der Dinge/Dienstleistungen/Daten) manifests itself here in the scenario of "Industrie 4.0" (in short: i4.0) - Production Society. The term "Industrie 4.0" describes the fourth industrial revolution (Figure 1) and is a partial aspect of the hightech strategy of the German Federal Government [1]. By vertical and horizontal networking of industrial structures new value added networks are created, which are able to still participate successfully in the global market in the future.

However, the fourth industrial revolution is distinguished from previously ones by the fact that it is not based on individual, seminal innovations. Rather "Industrie $4.0 "$ is characterised by the symbiosis of existing technologies. The focus here is among other things on applications in the fields of computer science, communication technology, sensor technology and actuator engineering. Nowadays, people are used to free access to information and a comprehensive networking in their private everyday life. Intense research is conducted to develop an approach to transmit such structures into industrial environment. The aim of the research is the realisation of the Internet of Things and cyber-physical Production Systems (CPPS). This should lead to a paradigm shift within the German industrial landscape and supersedes the hierarchical structure of the common automation pyramid (Figure 2) $[2,3]$.

CPPS consist of the sum of embedded systems, which capture their environment sensorially and are able to interact with actuators $[4,5]$. By connecting to digital 


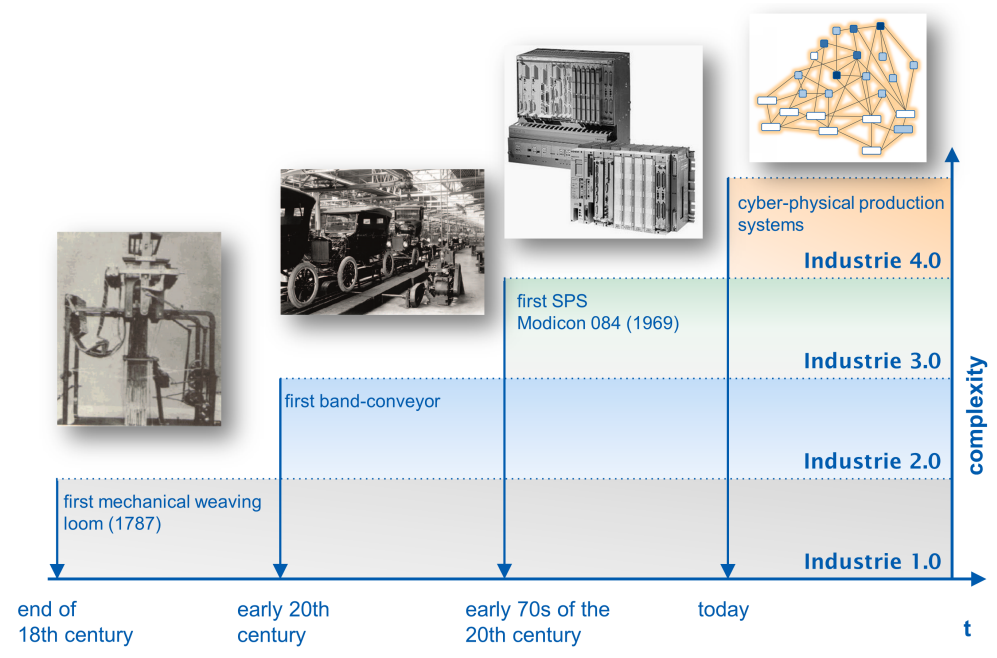

Figure 1: Overview of the different industrial revolutions

networks available data and services can be used. In addition an interface for human-machine communication is provided. An important point here is among other things the ability to collect, evaluate and provide data [6].

\section{cyber-physical Production Systems (CPPS)}

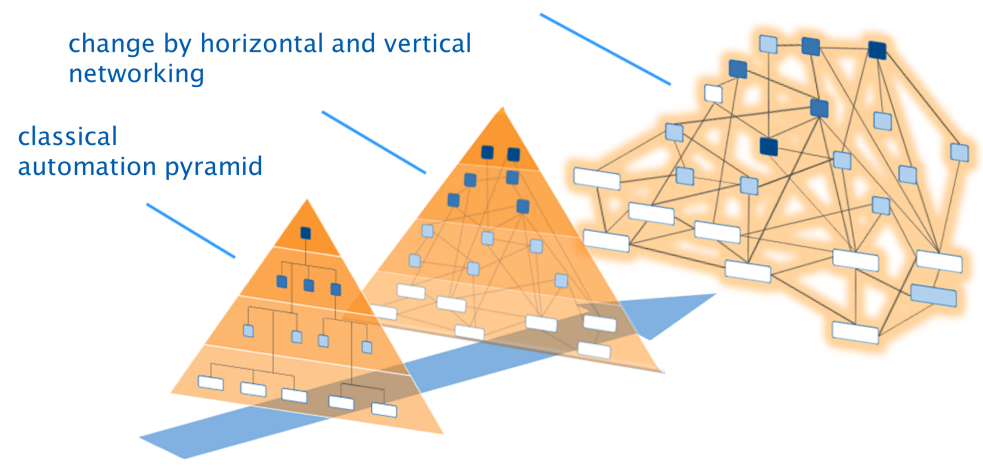

Figure 2: Turning the automation pyramid to a cyber-physical Production System

Diverse technical solutions to implement the Industry 4.0 approaches exist in subsisting or new production chains already. The main difficulties are to select sensors, actuators and network components from the available variety of solutions, to supplement the lack of technical entities and to assemble an overall system out of this. 
In the context of the project "Intelligent sensor systems for self-optimising production chains" (iSensPK) a research about the utilisation of existing sensor technology for cyber-physical Production Systems is carried out. A testing unit for force measurement on different gripper systems serves as a demonstrator.

Through the networking of sensors an intelligent environment recognition system is created. The exact image of the surrounding conditions should help to improve the results of force measurements. In addition the test conditions and results are minuted and recorded in the digital memory of the gripper. This data can then be queried by other entities in the production process. If a gripper is not able to meet the needs of the handling process parameters, such as the holding force as a function of the acceleration [7], the respective entity is able to refuse the use of the gripper. Due to this uncertain production procedures can be avoided.

\section{Market analysis of i4.0-capable components}

\subsection{Classification approach}

Guidelines and standards for the evaluation are in the origination process whether a technical solution copes with the requirement of the application i4.0. A first approach for an accordant classification results from the efforts of the VDI. An evaluation scheme, similar to the known electrical appliances IP protection classes, assesses a system or individual components according to their ability to communicate and their level of awareness in the information system (see Figure 3) [8].

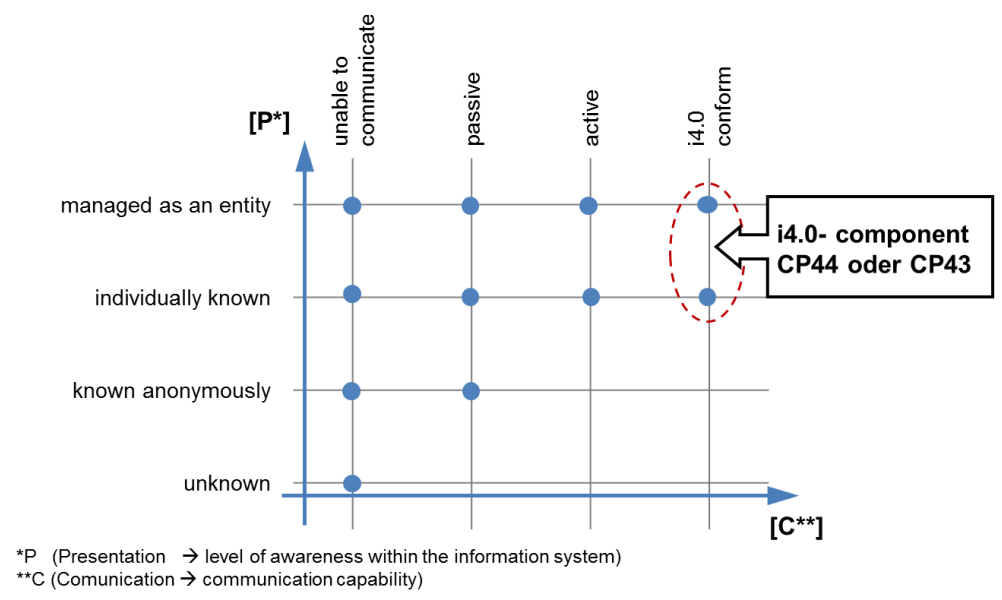

Figure 3: Classification approach for i4.0-components (according to $[8])$

Technical solutions with a Communication/Presentation(CP) index of CP43 or CP44 are defined as i4.0-components. i4.0-components can be particular parts, but also subsystems in which the individual components can only meet the requirements 
of the CP class as an assembly [8]. In the following the solutions available on the market that are suitable for the realization of i4.0-component will be discussed in more detail.

\subsection{Intelligent sensors}

The last generation of intelligent sensors which is represented on the market is also known as smart sensors. Such a component shall meet several requirements for a successful use in an i4.0 environment. During the connection of the sensor to the system it must automatically $\log$ on and needs to allow clear identification additionally. Through self-diagnosis the sensor should be able to spot faults and discontinuity in its performance and to display them automatically, too [9]. Supported by the localisation of the sensor on the human-machine interface a shortterm maintenance is possible. In case of failure, the storage of application specific parameters from the sensors ensure a 1:1 exchange due to the automatic checks of the automation system regarding the validity of the new sensor and setting of parameters. Equally corresponding settings can be stored and if required loaded for various production tasks. However, a major aspect is the decentralisation of intelligent functions with the result that sensors are taking part in the controlling process increasingly (i.e. to share relevant data for closed-loop process control). This procedure ensures that neither bandwidth nor computing capacity of the control is loaded with superfluous information that interferes with the process. It is also beneficial that no waiting time for a processing and feedback of the central control system is vital. In consequence, the minimum response times can be achieved necessary for high speed applications. Even the use of statistical data for calibration does not have to be renounced, because they can be deposited on the sensors. In an optimum case the controllers only have to evaluate a binary signal $[2]$.

\subsection{Communication technologies}

For the communication between sensors, actuators and control units, there are various interfaces and communication channels. Due to the increased use of sensors it is more and more important to build a reliable, collision-free network. Down on the field level sensors and actuators are usually connected to a bus system. The disadvantage of such a system is the incompatibility of the various systems to one another. Differences exist in physical specifications and various infrastructure components. Even sensors are often already designed for a specific bus system. Additionally, each sensor manufacturer has developed his own interface. The disadvantages which are resulting from variety and compatibility issues lead to two standardisation trends in the field of sensor technology. On one hand the use of a universal interface, where IO-Link looks promising. On the other hand the use of a standardised communication technology, whereby advantage could be taken of the pre-existing structure of the Ethernet. Thus the use of various wireless technologies is eased such as Wi-Fi or Bluetooth. 


\subsubsection{I/O-Link}

The interface currently used in sensor technology according to DIN EN 60947-5-2 [17] is no longer sufficient for today's sensor applications because they can transfer only one or two switching bits. Therefore many manufacturers have developed their own special interfaces. In consequence a multitude of cable variations can be found on the market, depending on which sensor is to be integrated [10]. The "USB standard for factory automation", as the I/O-Link, is called targets this point exactly. Initiated was the technology by Siemens through approaching several sensor manufacturers resulting in the establishment of the I/O-Link Consortium in 2010. In this context it was agreed to an interface with backward-compatible three-conductor physics. Consequently it is possible to operate both, traditional and highly complex sensors simultaneously in one system and to use for digital I/OLink signal transmission only a single unshielded three-wire power cable (Figure 4). Thus, all sensors and actuators are independent of the bus systems used on the control level, because they are only connected via point-to-point communication with the I/O-Link master. Further advantages consist in weight and material savings as well as lesser danger of cable break.
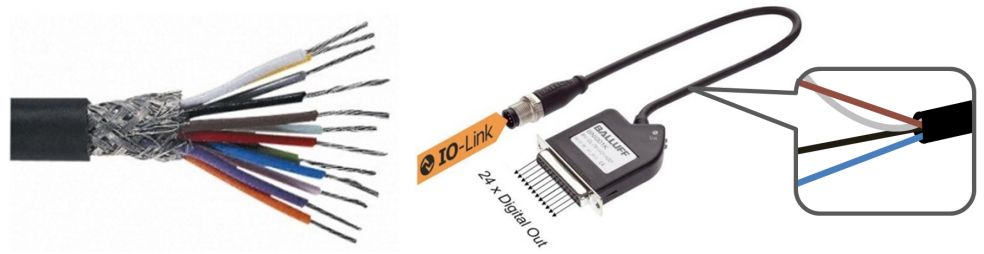

Figure 4: Compared to conventional cables (left) I/O-Link cables need less wires for the realisation of multiple digital outputs (right)

$[11,12]$

The in IEC 61131-9 [18] defined standard allows a less complex, bi-directional communication through which parameters can be set an transferred to subsequently added or identical sensors. In addition each I/O-Link component is clearly identifiable due to the I/O-DDs, the I/O-Link Device Description and able to independently $\log$ on a system with a complete description of its own functions. This is, together with the ability to communicate, one of the prerequisites for the future i4.0 sensor concept.

\subsubsection{Industrial Ethernet}

The open Ethernet protocol enables a direct, vertical integration. It has to be considered that the industrial Ethernet differs from the standard IEEE 802.3, as usually is used in office networks. About 30 different industrial Ethernet systems are in use worldwide. In most cases fieldbus manufacturers have written their own protocols. Some of the best known systems are PROFINET, POWERLINK, EtherNet / IP, EtherCAT and SERCOS III. The crucial factor is the difference to 
standard Ethernet, especially the real-time capability, i.e. the ability to transmit all data packets within a given time frame (hard real-time) or with a still permissible deviation (soft real time). In this context the achievable cycle times and clock jitter are around the tens to hundreds of milliseconds - at temperature measurements down to microseconds for digital control systems and motion control applications, see also [13]. The advantages of Ethernet are primarily the already standardised plug connections as well as the bigger range of available bandwidth in comparison to bus systems. Thus for example a PROFIBUS system (Siemens) is able to reach $12 \mathrm{Mbit} / \mathrm{s}$, while Ethernet is already moving in areas beyond the $1 \mathrm{Gbit} / \mathrm{s}$ barrier $[14]$.

\section{Demonstrator for cyber physical information re- trieval}

\subsection{Intelligent FRP manufacturing}

Components of fibre reinforced plastics (FRP) are essential for the production of wind power plants or the implementation of energy-efficient mobility concepts. For that reason automated manufacturing processes for FRP components have been developed particularly in the automotive and aerospace industries in recent years. These technologies are used, for instance, in the form of resin transfer moulding (RTM) in the manufacturing process of structural components for BMW electric vehicles $[15,9]$.

Just like other manufacturing processes the production of FRP parts is evaluated by the factors cost, time, quality and safety. Future production processes require a high degree of flexibility to cope with the aspects of Industrie 4.0. Considering the above-mentioned aspects the safe handling of flexible semi-finished products such as preforms creates a major barrier, preventing the automated production of FRP parts. A leverage point to overcome existing technology gaps can be found in the area of networking and self-optimising systems.

Through the integration of RFID tags in an FRP component it is possible to enable the component to provide information [16] about its condition itself. In addition it is achievable to store information for the handling process in the digital memory of the intelligent component. This information may be, the wrought material weight (corresponding to the processing phase), centre of gravity or specifications for surface finish. This way the component is able to interact with other entities of the handling process. Thus it is possible to request a gripper out of a pool of intelligent gripping tools, which has got the necessary properties, such as the holding force as a function of the acceleration to safely perform the desired operation (Figure 5). Inappropriate grippers are denied to accomplish the task by the according entity. This leads to greater safety and quality of the handling processes.

The gripping force parameters are stored in the memory of the gripper by use of RFID technology. Therefor previously performed gripping force measurements 


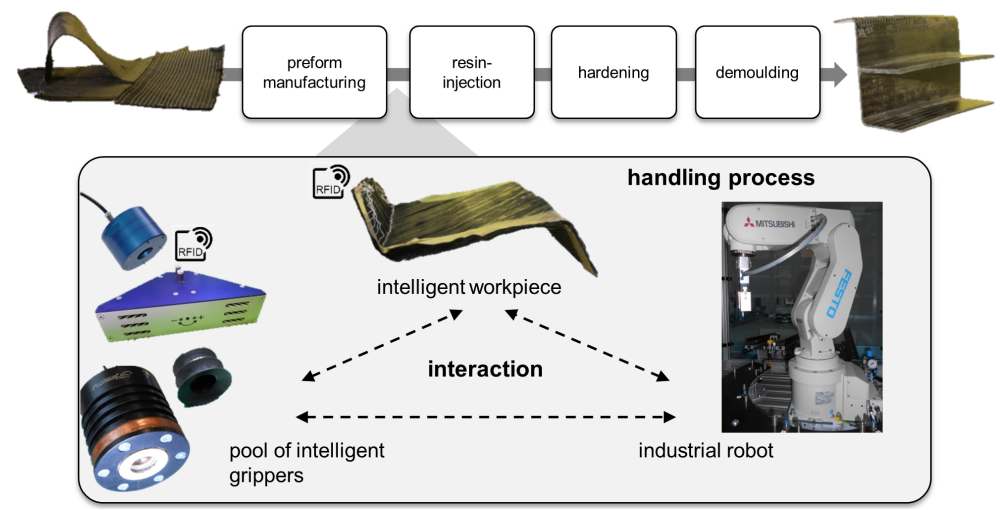

Figure 5: Handling process of an intelligent production of FRP parts

under consideration of disturbance variables within the field level are required.

\subsection{Experimental setup for sensor-assisted gripping force measurement}

As a result of the preliminary considerations different sensor technological entities are selected to be used within an experimental setup for cyber-physical information extraction. The demonstrator consists of a force and torque measurement within all six degrees of freedom for different gripper systems with simultaneous gathering of the environmental parameters. Taken into account are not only pressure, temperature and humidity values of the ambient air, but also pressures within the pneumatic gripping components or the temperature of the workpiece gripped by a cryo gripper. By comparing and evaluating the data the quality of the force measurement is meant to increase. A self-optimisation as well as a self-calibration procedure can be realized. Additionally, various communication interfaces come to use. That way the interchangeability of components are validated and advantages and disadvantages are highlighted. Figure 6 shows a digital mock-up of the planned construction.

The core of the construction consists of a single axis linear guide unit that can bring a force of up to $1000 \mathrm{~N}$ into the system. Due to off-centred force application multiaxial states of stress can be generated and observed as well. The testing facility is mobile so it can be used in various environments according to varying environmental conditions. For the force measurement different gripper systems are available. In addition to a two-finger gripper, cryo grippers for non-rigid sheet components can also be tested. These moisten the component with a thin layer of water and create a hydro adhesive connection by falling below the freezing point temperature. Depending on the position of the force measurement sensor, either mounted on the base plate or directly to the gripper unit, different quasi-static field 


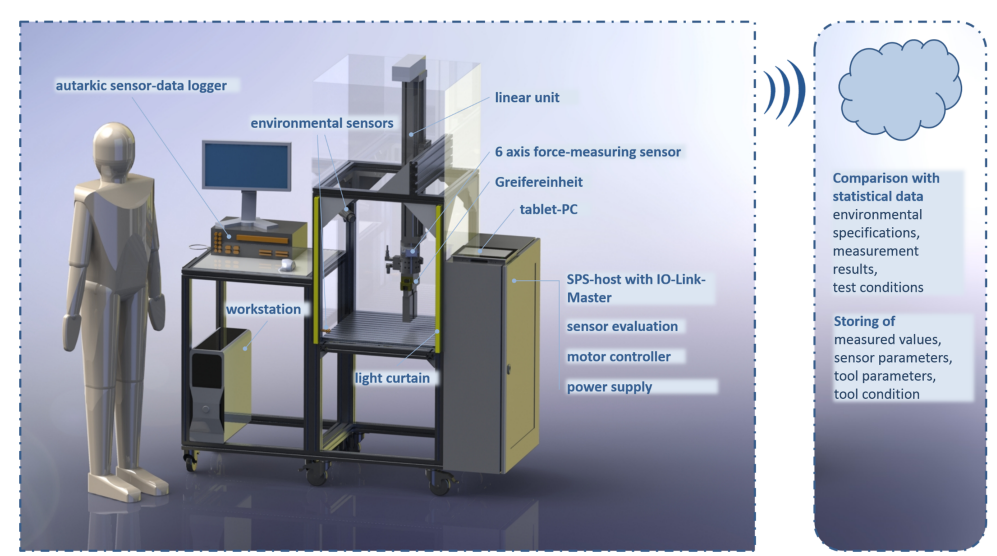

Figure 6: 3D model of the testing facility with explanation

conditions such as the shear-off of a component can be simulated. In further use it is conceivable to substitute the linear system with a robot unit to enable dynamic measurements of occurring accelerations during pivoting movements.

The central component of the force measuring system is a force measurement sensor, based on a silicon resistance strain gauge. The selection of an appropriate sensor considers a market analysis, regarding network suitability and resolution. Its outcome shows that the independent network application and integration of the sensor via Ethernet interface and MAC address in combination with a very fine resolution from 1.0 to $1.5 \%$ (depending on the axis) currently only can be realised by a force-measuring sensor. For the testing facility the model Schunk FT-85 Omega with a measuring range of up to $950 \mathrm{~N}$ and $40 \mathrm{Nm}$ is used (Figure $7)$.

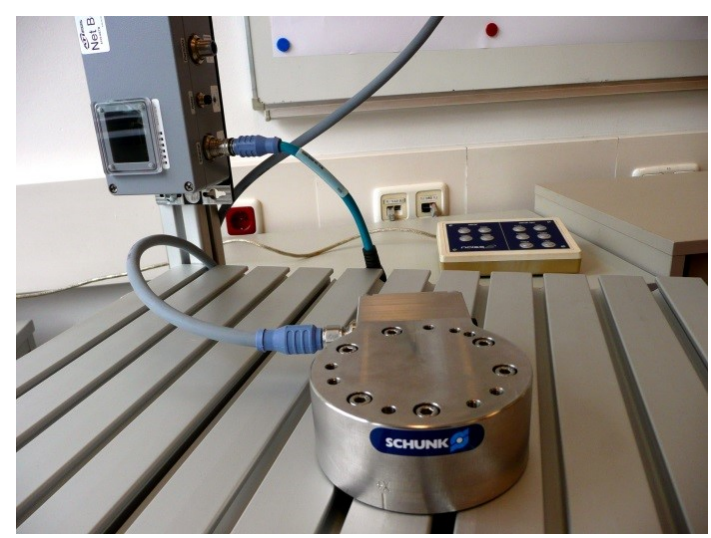

Figure 7: Photo of the force-measuring sensor FT-Omega85 
In order to show existing application possibilities of the underlying principle those are mapped onto a real application. One example is the monitoring of the state of utilised tools. This is done by saving measured values, results and protocols with the help of integrated RFID transponders. Thus the reading of current test results before the usage of a gripper is facilitated and allows the adaptation of process parameters, the possibility to keep spare parts ready and update process data. That way the achievable accuracy of a tool can be evaluated against the requirements of the manufacturing process. Before the commissioning potential operation deviations can be predicted and a decision about the processing release can be made. However not only at the machine, but also directly in the tool bearing a tool preselection is possible. For example tools with a high degree of wear and tear can still be used for jobs with lower quality requirement and safety standards. If a tool exceeds the preset limit of the tool parameters during an operation and calibration is no longer possible, this can be reported automatically and the tool can be disabled for usage. Due to constant monitoring the appropriate spare parts are already in stock some time before failure occurs.

\section{Conclusion and outlook}

Based on the studies to i4.0 entities a testing facility was designed for dynamic determination of actuator parameters in an industrial environment and a first mockup was created. Continually, after built up the facility experimental investigations of the communication paths will be performed. Based on the solutions, approaches are validated for intelligent networking of entities in the technical production environment. It is shown that the technical requirements for such a system already exist and only the realisation of the construction needs to be done. Further, possible uses are adressed in various industries, such as life science or plastics processing. Furthermore, as a partial project of the fourth industrial revolution, a contribution to the business location Germany is made. The objective is to turn the common hierarchical automation structures towards a cyber-physical Production System.

Through synthesis the structures used in the case study can be expanded to a larger application field. Thereby, it will be possible to increase the informational value of measurements through sensor data fusion and in consequence to improve spare parts management or production quality. Owing to the expansion of the test construction very complex structures can be represented with robot technology significantly, leading to a good basis for continuative projects. The application of different tools and newer sensors is possible and conceivable.

Acknowledgments. This paper is based upon the outcome of the WiTet-project (no: 80160682) "Aufbau und Entwick-lung von intelligenten Sensoriksystemen für selbstoptimierende Produktionsketten (iSensPK)". The project was co-funded with support from the "Europäischer Founds für regionale Entwicklung (EFRE)". 


\section{References}

[1] Kagermann, H.; Wahlster, W.; Helbig, J.: Umsetzungsempfehlungen für das Zukunftsprojekt Industrie 4.0: Abschlussbericht des Arbeitskreises Industrie 4.0., 2013

[2] Sauerer, J.: Smart Sensors, in: Workshop Sensorik für Erneuerbare Energien und Energie-effizienz, p. 18-24, 2013.

[3] Spath, D. (publ.): Produktionsarbeit der Zukunft - Industrie 4.0: Studie, Stuttgart: Fraunhofer-Verlag, 2013.

[4] Reinhart, G. et al: Cyber-Physische Produktionssysteme: Produktivitäts- und Flexibilitäts-steigerung durch die Vernetzung intelligenter Systeme in der Fabrik, in: wt Werkstattstechnik online, p. 84-89, 2013.

[5] Zhang, L.; Fallah, Y.; Jihene, R.: Cyber-Physical Systems: Computation, Communication, and Control, in: International Journal of Distributed Sensor Networks 2013, p. 1-2, abgeru-fen: 25.11.2014 url: http://dx.doi.org/10.1155/2013/475818, 2013.

[6] Vogel-Heuser, B. et al: CPS - eine Fallstudie: Eine Anwendung von CPS im Bereich der Wartung in der Produktautomatisierung, in: atp edition 55 , p. 50-56, 2013.

[7] Reiff-Stephan, J.: Probleme mit dem Greifen, in: A\&D Vorsprung Automation, p. 116-119, 2013.

[8] Epple, U. et al: Industrie 4.0 Statusreport: Gegenstände, Entitäten, Komponenten, Düssel-dorf: VDI/VDE, 2014.

[9] Reiff-Stephan, J.; von Lipinski, R.: Selbstoptimierende Prozesse als Schlüsseltechnologien zur Automatisierung der FVK-Produktion, in: Tagungsband AALE 2014, p. 379-382, Munich: Deutscher Industrieverlag GmbH, 2014.

[10] Kräußlich, W.: Sensorik und IO-Link als Wegbereiter für die Fabrik der Zukunft, abgerufen: 13.06.2014, url: ahttp://www.konstruktion.de/topstory/ sensorik-und-io-link-als-wegbereiter-fur-die-fabrik-der-zukunft/, 2013.

[11] CNC-kompakt (s.a.): Abbildung: Kabel 4-adrig geschirmt, abgerufen: 15.09.2014, url: http://www.cnc-kompakt.de/images/product_images/original_images/ kabel_geschirmt.jpg

[12] SPS-MAGAZIN (2011): Abbildung: Ventilinselstecker ersetzen aktorseitig die bisherige Parallelverdrahtung von Ventilinseln durch eine schlanke serielle IO-LinkVerbindung, abge-rufen: 15.09.2014, url: http://www.sps-magazin.de/bilder/ artikel/77879.jpg

[13] Meindl, A.: Ultraschnelle Automatisierung: reACTION Technology, in: Automotion edition 5-2014, p. 3-6, 2014.

[14] Lachello, L. et al: Systemvergleich: Die 5 wesentlichen Systeme, in: Industrial Ethernet Facts, edition 2-2013, retrieved: 27.08.2014, url: http://www .ethernet-powerlink.org/fileadmin/user_upload/Dokumente/ Dokumente/EPSG_IEF2ndEdition_d_Web.pdf

[15] Dix, M.; Beck, S.; Repper, A.; Hinterzölzl, R.: Integrierte Materialmodellierung für CFK-Prozesssimulation. in: lightweightdesign, edition 5-2013, Springer-Verlag $\mathrm{GmbH}$, Heidelberg

[16] Mayordomo, M.: Durchgängige Überwachung mit RFID für Windparks. in: RFID im Blick, edition 10-2012, Düsseldorf, Verlag \& Freie Medien Anja Van Bocxlaer 
[17] DIN EN 60947-5-2: Niederspannungsschaltgeräte - Teil 5-2: Steuergeräte und Schaltele-mente - Näherungsschalter (IEC 60947-5-2).

[18] DIN EN 61131-9: Speicherprogrammierbare Steuerungen - Teil 9: Schnittstelle für die Kommunikation mit kleinen Sensoren und Aktoren über eine Punkt-zu-PunktVerbindung (IEC 65B/815/CDV). 